\section{La faim du temps !}

\section{The hunger of time!}

\section{B. Dahan $\cdot$ A. Eche $\cdot$ B. Renaud}

Reçu le 9 octobre 2013 ; accepté le 26 janvier 2014

(C) SFMU et Springer-Verlag France 2014

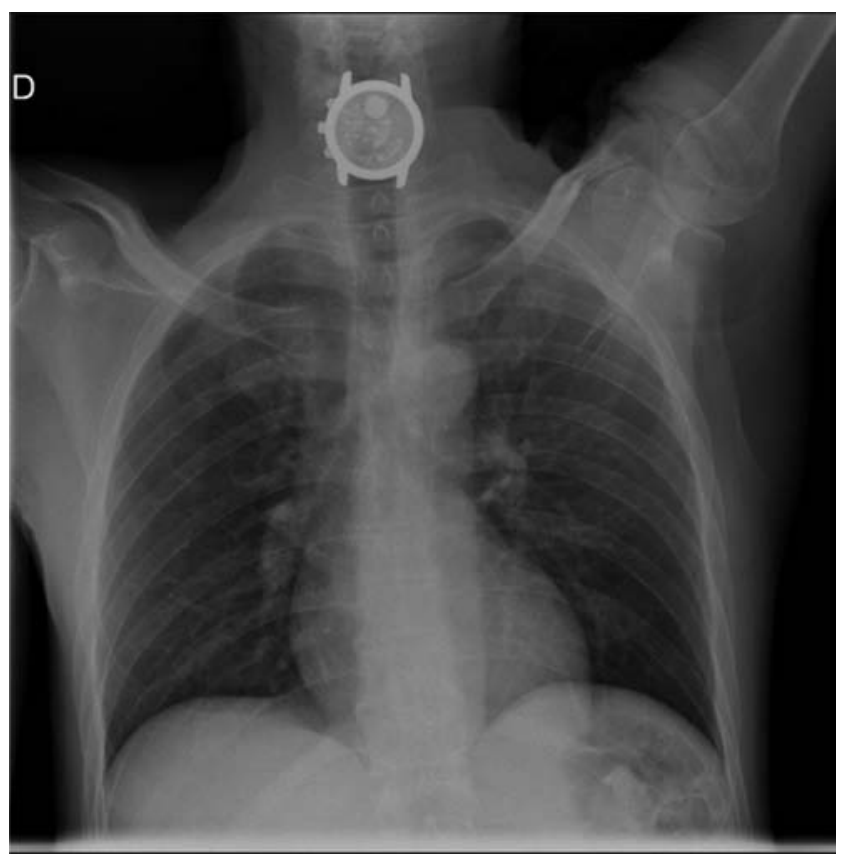

Fig. 1 Radiographie pulmonaire de face

Un patient âgé de 51 ans est amené par les forces de l'ordre aux urgences, dans le cadre d'une garde à vue, pour ingestion de corps étranger. Le patient, eupnéique à l'arrivée, se plaint d'une odynophagie. L'examen buccopharyngé montre une discrète hypersialorrhée. Le patient ne présente pas de signes cliniques évocateurs d'une perforation de l'œsophage (emphysème sous-cutané cervical, raideur de nuque, dyspnée). La radiographie pulmonaires de face (Fig. 1) met en évidence la présence d'un corps étranger radio-opaque correspondant à une montre à quartz en projection de l'œsophage cervical.

Une fibrolaryngoscopie en urgence est proposée au patient. Devant le refus de ce dernier, la décision de surveillance en hospitalisation est prise et le patient est laissé à jeun. Neuf heures après son admission, à l'occasion d'un vomissement la montre est évacuée. Le patient récidive alors son geste en ingérant sa brosse à dent, la radiographie de contrôle est normale. À l'inverse des montres, les brosses à dents ne sont pas radio-opaques. La prise en charge d'ingestion de corps étranger nécessite la recherche de signes de complications ; dans ce cas, celle d'une perforation de l'œsophage ou d'une ulcération due à l'altération de la pile bouton (présence probable de lithium) [1,2].

\section{Références}

1. Ikenberry SO, Jue TL, Anderson MA, et al (2011) ASGE Standards of Practice Committee, Management of ingested foreign bodies and food impactions. Gastrointest Endosc 73:1085-91

2. Letard J, Gay G, Ponchon T, et al (2004) Recommandations de la Société française d'endoscopie digestive. Les corps étrangers ingérés, http://www.sfed.org (dernier accès le 24 janvier 2014)

B. Dahan $(\bowtie) \cdot$ A. Eche $\cdot$ B. Renaud

Service des urgences, Smur, hôpital Cochin,

Assistance publique-hôpitaux de Paris,

27, rue du faubourg Saint-Jacques,

F-75014 Paris, France

e-mail : benjamin.dahan@inserm.fr

Université Paris Descartes,

12, rue de l'École de médecine,

F-75006 Paris, France 